\title{
Resveratrol attenuates matrix metalloproteinase-9 and -2- regulated differentiation of HTB94 chondrosarcoma cells through the p38 kinase and JNK pathways
}

\author{
EUN JEONG GWEON and SONG-JA KIM
}

\author{
Department of Biological Sciences, College of Natural Sciences, Kongju National University, \\ Chungnam 314-701, Republic of Korea
}

Received November 21, 2013; Accepted January 7, 2014

DOI: $10.3892 /$ or.2014.3192

\begin{abstract}
Resveratrol has been shown to possess anticancer, anti-aging, and anti-inflammatory properties. Matrix metalloproteinases (MMPs) appear to be responsible for much of the extracellular matrix (ECM) degradation observed in the progression of cancer, aging and inflammation. We found that resveratrol significantly inhibited MMP-2 and MMP-9, and induced the expression of type II collagen and sex-determining region Y-box (SOX)-9 and the production of sulfated proteoglycans in HTB94 chondrosarcoma cells. Moreover, inhibition of MMPs with an MMP inhibitor further enhanced the effects of resveratrol. Phosphorylation of p38 was increased and phosphorylation of c-Jun N-terminal kinase (JNK) was inhibited by resveratrol. Treatment with SB203580, a p38 kinase inhibitor, enhanced the suppression of MMP-2 and MMP-9 by resveratrol and inhibited resveratrol-induced stimulation of type II collagen and SOX-9 expression and production of sulfated proteoglycans. Treatment with SP600125, a JNK inhibitor, attenuated the effects of resveratrol on MMP-2 and MMP-9, and accelerated resveratrol-induced effects on type II collagen, SOX-9 and sulfated proteoglycan production. Our results suggest that resveratrol inhibits MMP-induced differentiation via the p38 kinase and JNK pathways in HTB94 chondrosarcoma cells.
\end{abstract}

\section{Introduction}

Chondrosarcoma is a type of bone cancer originating from cartilaginous tissue and is the second most common bone tumor (1). Chondrosarcomas respond poorly to radiotherapy and chemotherapy, which are the currently used treatment strategies for this malignancy, making the management of chondrosarcomas a challenge (2). Chondrosarcoma accounts

Correspondence to: Professor Song-Ja Kim, Department of Biological Sciences, College of Natural Sciences, Kongju National University, 182 Singwan-dong, Gongju 314-701, Republic of Korea E-mail: ksj85@kongju.ac.kr

Key words: resveratrol, matrix metalloproteinases, differentiation for $25-30 \%$ of all cancers originating from the skeletal system (3). Osteoarthritis (OA), the most common joint disease, is a functionally debilitating condition characterized by degeneration of articular cartilage. Intrinsic resorption occurs when chondrocytes, the only cells present in the articular cartilage, over-exert their capacity for stimulating resorption of the extracellular matrix (ECM) (4,5). Articular chondrocytes maintain a dynamic equilibrium between synthesis and degradation of ECM components, including type II collagen fibrils surrounding and restraining large hydrated aggregates of proteoglycans, allowing normal cartilage to function as a shock absorber and withstand compressive loads (5). ECM degradation and remodeling require the action of extracellular proteinases, of which matrix metalloproteinases (MMPs) have been shown to play a significant role (6). MMPs are a family of zinc-dependent proteinases that facilitate the breakdown and turnover of cartilage, and their levels are elevated in joint tissues of patients with OA and rheumatoid arthritis (RA) $(7,8)$.

Resveratrol (trans-3,4',5-trihydroxystilbene) is a natural polyphenolic compound present in various types of plants, such as grapes and in food products. Its powerful and diverse biological effects have been well documented in recent years (5). Resveratrol exhibits many biological activities, including anticancer, anti-inflammatory, anti-aging, anti-oxidant, antibacterial, antifungal and antiviral effects (9). Resveratrol has been shown to inhibit MMPs via modulation of sirtuin-1 (SIRT-1) in human fibrosarcoma cells. Notably, the activation of SIRT-1 in the presence of resveratrol specifically inhibits the expression of MMP-9 in HT1080 cells (10). Recent studies have demonstrated the chondroprotective effects and mechanisms of resveratrol in chondrocytes stimulated with advanced glycation end products, including a previous study, in which we delineated the mechanisms underlying the resveratrolmediated protection of cartilage from advanced glycation end product-induced damage, and addressed the potential therapeutic benefit of resveratrol in the treatment of OA (11). Another recent study focused on the effect of resveratrol on metastasis in 4T1 mouse breast cancer cells. Resveratrol inhibited cancer metastasis both in vitro and in vivo, and this inhibition was likely due to the reduction in MMP-9 activity caused by resveratrol (12). Moreover, resveratrol reduced lung adenocarcinoma cell metastasis by suppressing heme 
oxygenase (HO)-1-mediated nuclear factor (NF)- $\kappa$ B pathway activation and subsequently downregulated the expression of MMPs. Resveratrol inhibited the transcription-activator function of HO-1 and subsequently MMP-2 and MMP-9 expression in human lung cancer cells as well. The inhibitory effects of resveratrol on MMP expression and invasion of lung cancer cells were, in part, found to be associated with its regulation of the HO-1-mediated effects on the NF- $\mathrm{B}$ pathway (13).

The mitogen-activated protein kinase (MAPK) pathway is an evolutionarily conserved kinase pathway that links extracellular signals to the machinery that controls fundamental cellular processes such as proliferation, differentiation, invasion and apoptosis in various types of cells, including cancer cells (14). Among the MAPK subtypes, the extracellular signal-regulated kinases (ERKs) are activated in response to stimulation with mitogen or growth factor, whereas JNK and p38 kinase are regulated under conditions of cellular stress (15). A recent study demonstrated that induction of MMP-2 and MMP-9 involves multiple signaling cascades, particularly of the MAPK pathway (16). Other studies showed that epidermal growth factor receptor (EGFR) regulates MMP function in fibroblasts through the MAPK and AP-1 pathways (17) and that transforming growth factor (TGF)- $\beta$-induced transcriptional activation of MMP-2 is mediated by activating transcription factor (ATF)-2 in human breast epithelial cells (18).

However, the molecular mechanism of MMP-regulated differentiation of human chondrosarcoma cells by resveratrol remains largely unclear. Therefore, in the present study, we sought to investigate the mechanism through which resveratrol regulates MMP-mediated differentiation, using HTB94 human chondrosarcoma cells as a model. We also sought to identify the underlying signaling pathways involved in this process. Our results indicate that resveratrol suppresses MMP-regulated differentiation through its effects on the p38 kinase and JNK pathways in HTB94 human chondrosarcoma cells.

\section{Materials and methods}

Materials. Resveratrol (trans-3,4',5-trihydroxystilbene) was obtained from Sigma Aldrich (St. Louis, MO, USA). Dulbecco's modified Eagle's medium (DMEM) and fetal bovine serum (FBS) were purchased from Invitrogen (Burlington, ON, Canada). Streptomycin and penicillin were purchased from Sigma Aldrich. SB203580, SP600125 and MMP inhibitor III were obtained from Calbiochem (San Diego, CA, USA). Antibodies specific for MMP-2 (Santa Cruz Biotechnology, Santa Cruz, CA, USA), MMP-9 (Santa Cruz Biotechnology), type II collagen (Santa Cruz Biotechnology), SOX-9 (Santa Cruz Biotechnology), phosphorylated (p)p38 (Cell Signaling Technology, Danvers, MA, USA), p38 (Santa Cruz Biotechnology), pJNK (Cell Signaling Technology), JNK (Santa Cruz Biotechnology) and actin (Santa Cruz Biotechnology) were used for the experiments.

Cell lines and culture. The HTB94 (human chondrosarcoma) cell line was purchased from the American Type Culture Collection (ATCC; Rockville, MD, USA). HTB94 cells were maintained in DMEM containing $10 \% \mathrm{FBS}, 50 \mu \mathrm{g} / \mathrm{ml}$ streptomycin and $50 \mathrm{U} / \mathrm{ml}$ penicillin. Cells were maintained at $37^{\circ} \mathrm{C}$ in a humidified atmosphere with $5 \% \mathrm{CO}_{2} . \mathrm{HTB} 94$ cells were then plated on culture dishes at a density of $0.9 \times 10^{5}$ cells/dish, and the cells were allowed to reach $70 \%$ confluency before they were treated with inhibitors or used in the experiments.

Viability assay (MTT). HTB94 cells were plated at a density of $0.9 \times 10^{5}$ cells/well on 96-well plates and incubated overnight, and the medium was replaced with fresh medium on the following day. The cells were treated with various concentrations of resveratrol or left untreated, in the absence or presence of SB203580, SP600125 or MMP inhibitor III for $24 \mathrm{~h}$. After these treatments, $10 \mu \mathrm{l}$ of MTT reagent I (methyl thiazole tetrazolium) $(10 \mathrm{mg} / \mathrm{ml})$ was added to each well. After incubating the cells for another $24 \mathrm{~h}, 100 \mu \mathrm{l}$ of MTT reagent II [solubilization buffer, $10 \%$ SDS with $0.01 \mathrm{~N} \mathrm{HCl}$ in dimethylsulfoxide (DMSO)] was added to each well, and the cells were incubated overnight at $37^{\circ} \mathrm{C}$. Finally, the absorbance of the samples was measured at $595 \mathrm{~nm}$ by using an enzyme-linked immunosorbent assay (ELISA) plate reader.

Gelatin zymography. HTB94 cells were seeded on a 35-mm tissue culture dish and kept overnight for attachment. The next day, the medium was replaced with DMEM containing 2\% FBS, and the cells were incubated overnight at $37^{\circ} \mathrm{C}$. HTB94 cells were treated with the indicated reagents in DMEM containing $2 \%$ FBS. The cells were lysed to prepare protein extracts, and equal amounts of total protein were resolved by sodium dodecyl sulfate (SDS)-polyacrylamide gel electrophoresis (PAGE) on $7.5 \%$ polyacrylamide gels containing $0.1 \%$ gelatin. After SDS-PAGE, the gels were washed with $2.5 \%$ Triton X-100 for $90 \mathrm{~min}$ and incubated with gelatin incubation buffer ( $5 \mathrm{mM} \mathrm{CaCl}_{2}, 0.2 \mathrm{M} \mathrm{NaCl}$ and $50 \mathrm{mM}$ Tris) for 20-24 h. Next, the gels were stained with Coomassie blue solution for 60-90 min and destained with a solution containing $30 \%$ acetic acid and $10 \%$ methanol. The bands were quantified by densitometric analysis using the ImageJ software package.

Western blot analysis. HTB94 cells grown in 35-mm tissue culture dishes were treated with the indicated reagent, harvested and washed with cold phosphate-buffered saline (PBS). Proteins were extracted using cold radioimmunoprecipitation assay lysis buffer $[50 \mathrm{mM}$ Tris- $\mathrm{HCl}(\mathrm{pH} 7.4)$, $150 \mathrm{mM} \mathrm{NaCl}, 1 \%$ Nonidet P-40, 0.1\% SDS, supplemented with protease inhibitors $(10 \mu \mathrm{g} / \mathrm{ml}$ aprotinin, $10 \mu \mathrm{g} / \mathrm{ml}$ pepstatin, $10 \mu \mathrm{g} / \mathrm{ml}$ leupeptin, and $1 \mathrm{mM} 4$-(2-aminoethyl) benzenesulfonyl fluoride) and phosphatase inhibitors (1 $\mathrm{mM}$ $\mathrm{NaF}$ and $1 \mathrm{mM}$ sodium orthovanadate)]. Equal amounts of total cellular proteins were resolved by SDS-PAGE, and the proteins were transferred to nitrocellulose membranes (Whatman Schleicher and Schuell, Dachen, Germany). The membranes were blocked with $5 \%$ non-fat dry milk in Trisbuffered saline (TBS). The membranes were incubated with antibodies specific for MMP-2, MMP-9, type II collagen, SOX-9, pp38, p38, pJNK, JNK and actin overnight at $4^{\circ} \mathrm{C}$. The membranes were than washed with TBST (TBS containing Tween-20) and incubated with horseradish peroxidaseconjugated secondary antibodies (Sigma Aldrich) for $2 \mathrm{~h}$. The bands were quantified by densitometric analysis using the ImageJ software package. 
A

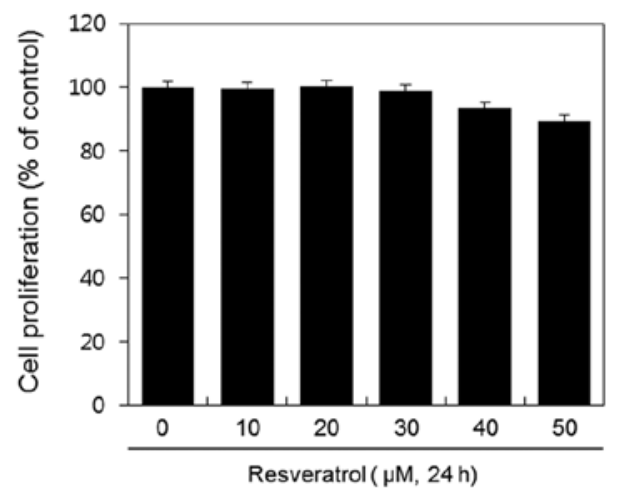

C

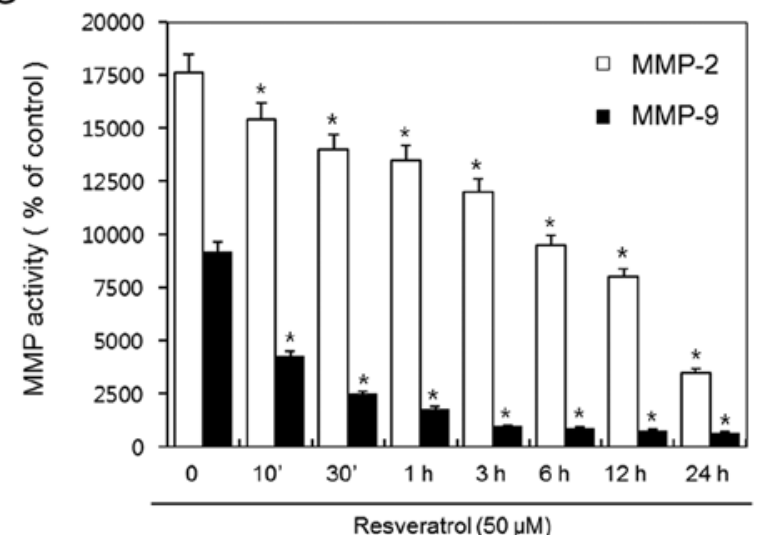

B

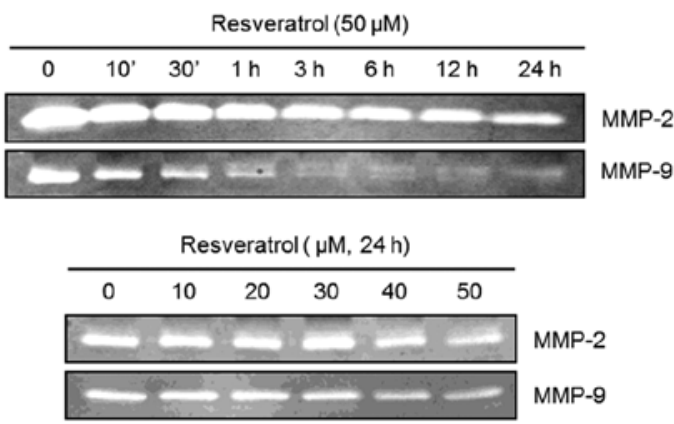

D

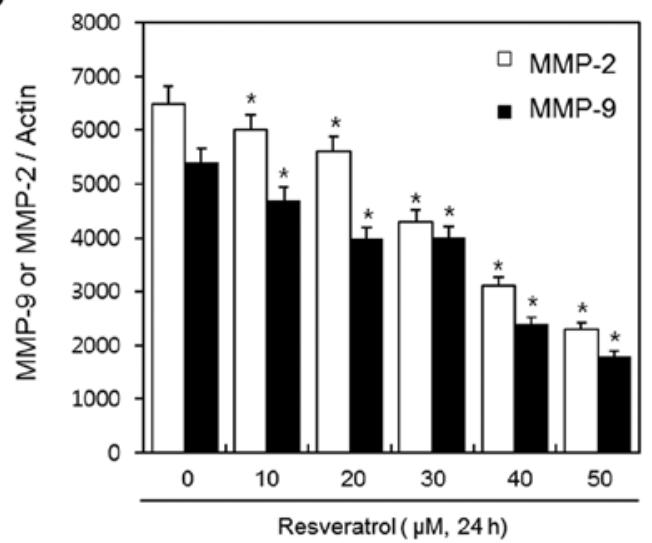

Figure 1. Effects of resveratrol on the activation of MMP-2 and MMP-9 in HTB94 cells. HTB94 cells were untreated (control) or treated with the various indicated concentrations of resveratrol for $24 \mathrm{~h}$. (A) Cell proliferation was measured using MTT assay. HTB94 cells were untreated (control) or treated with $50 \mu \mathrm{M}$ of resveratrol for the indicated time periods or with the various indicated concentrations of resveratrol for $24 \mathrm{~h}$. (B) Activation of MMP-2 and MMP-9 was detected using gelatin zymography assay. (C and D) The relative amounts of MMP-2 and MMP-9 were quantified by densitometric measurement (ImageJ). Statistically significant differences were noted between the control and the treatment groups. These data are the results of a typical experiment. ${ }^{*}<0.05$ compared to the control.

Alcian blue staining. HTB94 cells were fixed with $95 \%$ methanol at $-20^{\circ} \mathrm{C}$ for $5 \mathrm{~min}$. Next, the cells were stained with $0.1 \%$ Alcian blue in $0.1 \mathrm{M} \mathrm{HCl}$ overnight. The cells were washed 3 times with PBS and incubated with $6 \mathrm{M}$ guanidine $\mathrm{HCl}$ for $6 \mathrm{~h}$. Production of sulfated proteoglycans was evaluated by measuring the absorbance of the stained cells at $620 \mathrm{~nm}$ using an ELISA plate reader.

Immunofluorescence (IF) analysis. HTB94 cells were fixed with cold $3.5 \%$ paraformaldehyde in PBS for $20 \mathrm{~min}$ at room temperature. Cells were permeabilized in PBS containing $0.1 \%$ Triton X-100 for $15 \mathrm{~min}$ at room temperature. The fixed cells were subsequently washed with PBS and incubated for $2 \mathrm{~h}$ with antibodies against MMP-9 and type II collagen. Then, the cells were washed with PBS and incubated with secondary antibodies for $1 \mathrm{~h}$, after which the cells were washed again and incubated for $15 \mathrm{~min}$ with DAPI (Invitrogen). The cells were washed 3 times with PBS and observed under a fluorescence microscope.

Statistical analysis. The results are expressed as the means \pm standard deviation (SD). The significance of differences between experimental and control groups was assessed using one-way analysis of variance (ANOVA). Significant differences were defined at the level of $\mathrm{P}<0.05$.

\section{Results}

Resveratrol reduces activation of MMPs in human chondrosarcoma HTB94 cells. To determine the cytotoxic effects of resveratrol on HTB94 cells, we treated cells with 10, 20, 30, 40 and $50 \mu \mathrm{M}$ of resveratrol for $24 \mathrm{~h}$ and evaluated the cell viability using the MTT assay. The results showed that treatment with resveratrol did not affect the viability of HTB94 cells (Fig. 1A). Next, HTB94 cells were left untreated (control) or treated with $50 \mu \mathrm{M}$ resveratrol for the indicated time periods or treated with the indicated concentrations of resveratrol for $24 \mathrm{~h}$. The activation of MMP-2 and MMP-9 was then analyzed using gelatin zymography. Resveratrol markedly reduced activation of MMP-2 and MMP-9 in a time- and dose-dependent manner (Fig. 1B). The zymography data were quantified by densitometric analyses using ImageJ (Fig. 1C and D). These results suggest that resveratrol reduces activation of MMP-2 and MMP-9 in HTB94 cells.

Resveratrol induces differentiation in human chondrosarcoma HTB94 cells. HTB94 cells were left untreated (control) or treated with $50 \mu \mathrm{M}$ of resveratrol for the indicated time periods or treated with the indicated various concentrations of resveratrol for $24 \mathrm{~h}$. The expression of type II collagen, SOX-9 and actin was detected using western blotting. Resveratrol induced 
A

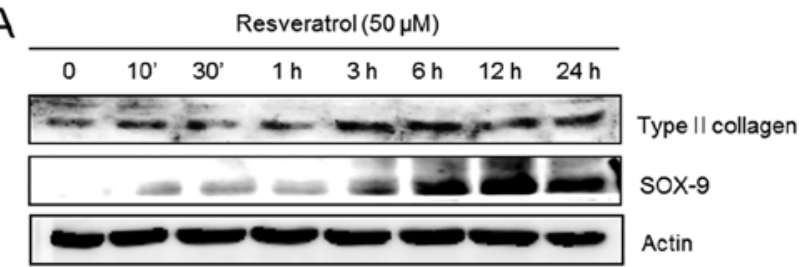

C

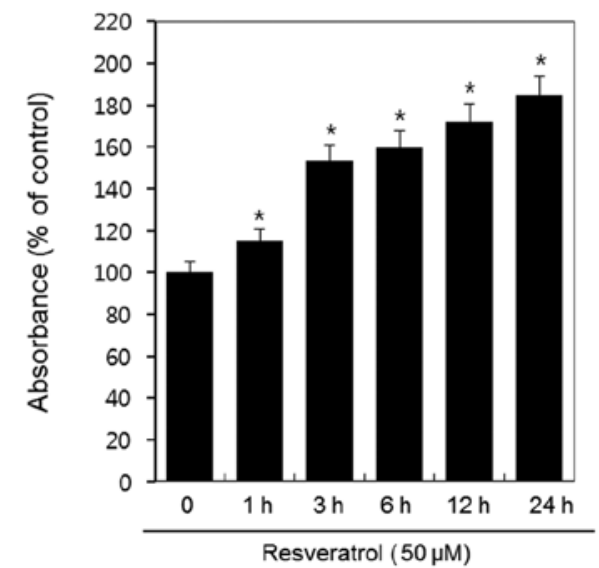

B

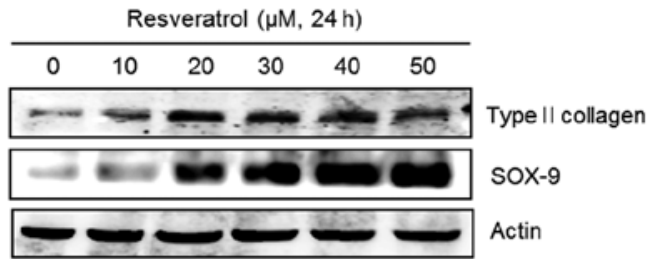

D

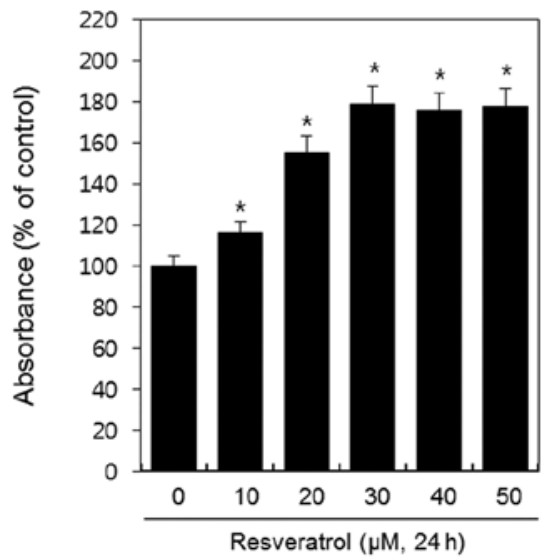

Figure 2. Effects of resveratrol on the differentiation of HTB94 cells. HTB94 cells were untreated (control) or treated with $50 \mu \mathrm{M}$ of resveratrol for the indicated time periods or with various indicated concentrations of resveratrol for $24 \mathrm{~h}$. (A and B) Expression of type II collagen, SOX-9 and actin were detected using western blot analysis. Actin was used as loading control. (C and D) Accumulation of sulfated proteoglycans was determined by Alcain blue staining. Statistically significant differences were noted between the control and the treatment groups. These data are the results of a typical experiment. ${ }^{*} \mathrm{P}<0.05$ compared to the control.

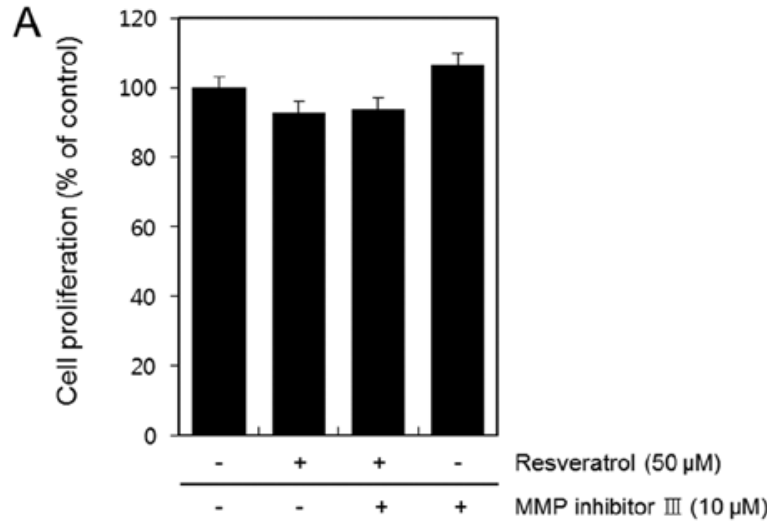

C

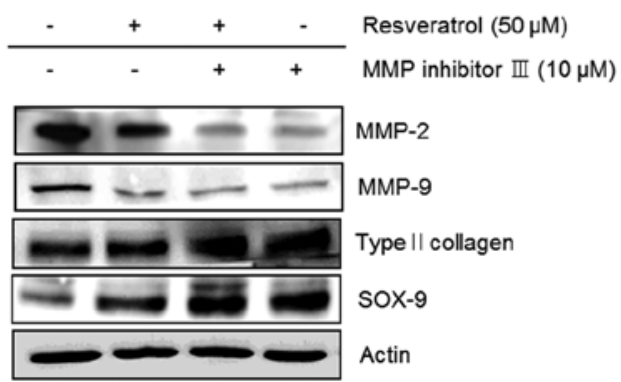

B

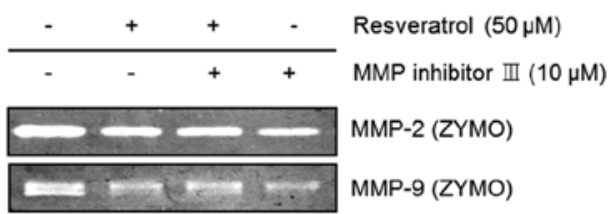

D

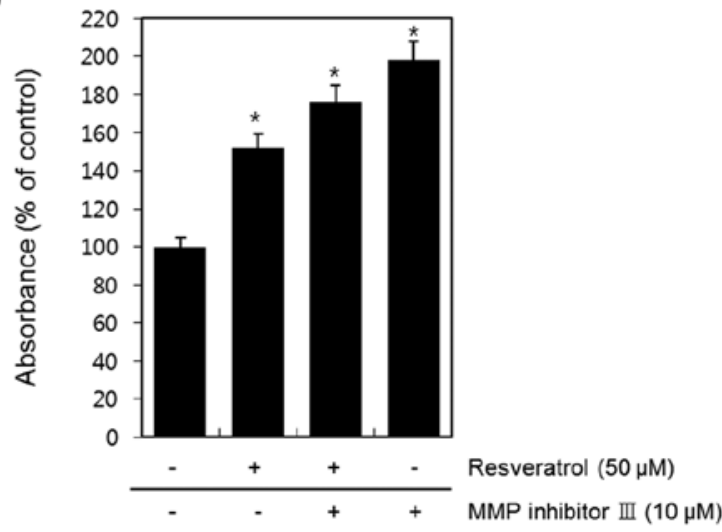

Figure 3. Effects of resveratrol on the MMP-regulated differentiation of HTB94 cells. HTB94 cells were untreated (control) or treated with resveratrol in the absence or presence of $10 \mu \mathrm{M}$ MMP inhibitor III for $24 \mathrm{~h}$. (A) Cell proliferation was measured using MTT assay. (B) Activation of MMP-2 and MMP-9 was detected using gelatin zymography assay. (C) Expression of MMP-2, MMP-9, type II collagen, SOX-9 and actin was detected using western blot analysis. Actin was used as the loading control. (D) Accumulation of sulfated proteoglycans was determined by Alcain blue staining. Statistically significant differences were noted between the control and the treatment groups. These data are the results of a typical experiment. " $\mathrm{P}<0.05$ compared to the control. 
A

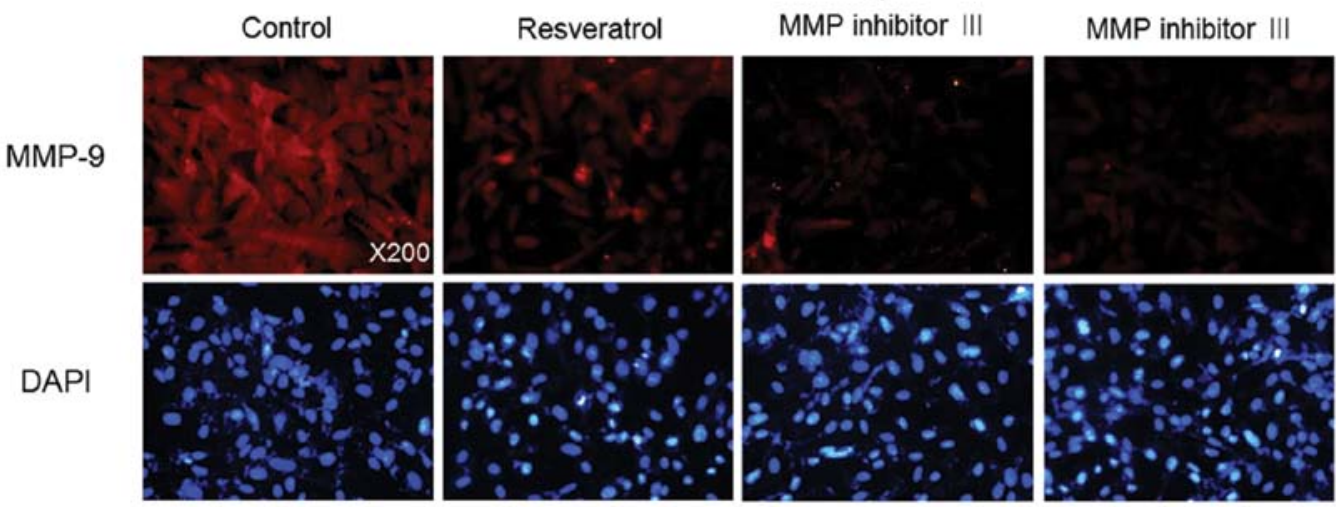

B

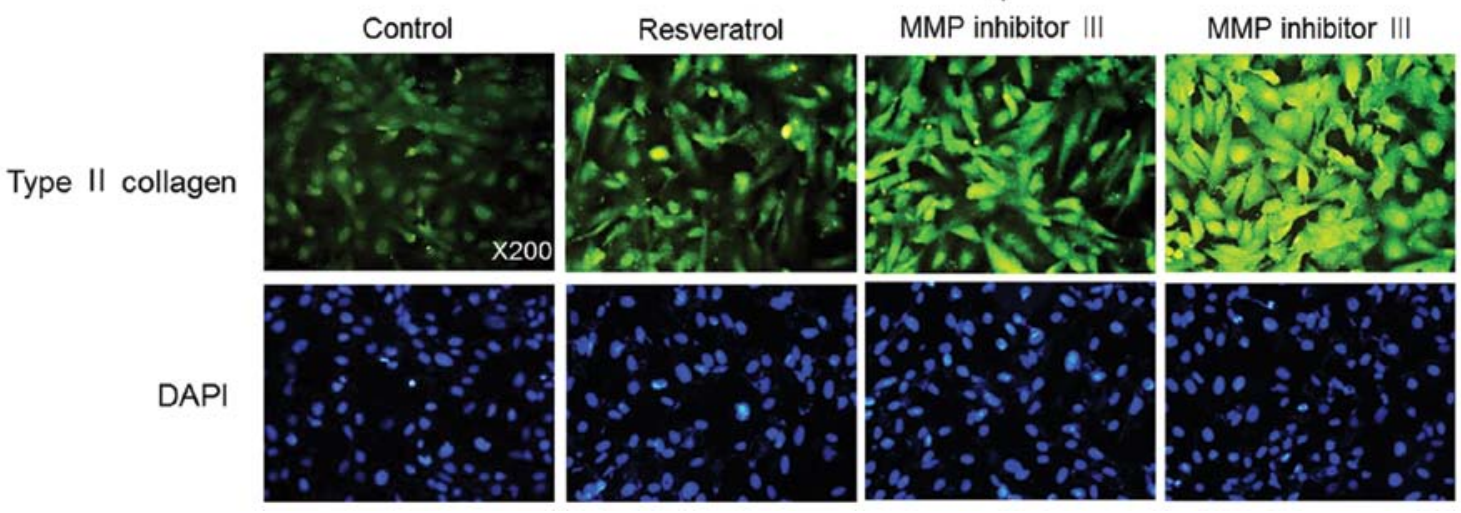

Figure 4. Effects of resveratrol on MMP-9-mediated regulation of type II collagen expression in HTB94 cells. HTB94 cells were untreated (control) or treated with resveratrol in the absence or presence of $10 \mu \mathrm{M}$ MMP inhibitor III for $24 \mathrm{~h}$. (A) Expression of MMP-9 and (B) type II collagen was detected by immunofluorescence staining. Statistically significant differences were noted between the control and the treatment groups . These data are the results of a typical experiment.

a significant increase in the expression of type II collagen and SOX-9 in a time- and dose-dependent manner (Fig. 2A and B). Production of sulfated proteoglycans was analyzed using Alcian blue staining. Consistent with the expression patterns of type II collagen, resveratrol induced the production of sulfated proteoglycans (Fig. 2C and D). These data suggest that resveratrol increases expression of type II collagen and SOX-9 and the production of sulfated proteoglycan in HTB94 chondrosarcoma cells. Taken together, these results indicate that resveratrol induces differentiation in HTB94 cells.

Resveratrol-induced differentiation occurs via its effects on MMPs. We left HTB94 cells untreated (control) or treated them with resveratrol in the absence or presence of $10 \mu \mathrm{M}$ MMP inhibitor III for $24 \mathrm{~h}$. To evaluate the cytotoxic effects on cell viability, we performed an MTT assay. The results indicated that co-treatment with resveratrol and MMP inhibitor III did not affect the viability of HTB94 cells (Fig. 3A). Activation of MMP-2 and MMP-9 was assessed using gelatin zymography. We found that MMP inhibitor III suppressed the activity of MMP-2 and MMP-9, and that the extent of this suppression was greater in the presence of resveratrol (Fig. 3B). Expression of MMP-2, MMP-9, type II collagen, SOX-9 and actin was assessed by western blot analysis. Inhibition of MMP-2 and MMP-9 with MMP inhibitor III further increased the resveratrol-induced expression of type II collagen and SOX-9 (Fig. 3C). Consistent with the expression patterns of type II collagen, inhibition of MMP-2 and MMP-9 with MMP inhibitor III further enhanced the resveratrol-induced production of sulfated proteoglycans (Fig. 3D). Moreover, consistent with the western blotting data, immunofluorescence analysis showed that treatment with MMP inhibitor III enhanced the resveratrol-mediated suppression of MMP-9 expression and increase in type II collagen expression (Fig. 4).

These data suggest that resveratrol decreases MMP-2- and MMP-9-regulated expression of type II collagen and SOX-9, and the production of sulfated proteoglycan in HTB94 chondrosarcoma cells. Taken together, our results indicate that resveratrol reduces MMP-regulated differentiation in HTB94 cells.

Resveratrol reduces MMP-regulated differentiation via p38 kinase and JNK pathways in HTB94 cells. We sought to identify the upstream signaling pathways involved in the resveratrol-mediated suppression of MMP-regulated differentiation in chondrosarcoma cells. HTB94 cells were left untreated (control), treated with $50 \mu \mathrm{M}$ of resveratrol for the indicated time periods or treated with the various concentrations of resveratrol for $24 \mathrm{~h}$. The expression of pp38, p38, pJNK, JNK and actin was analyzed using western blotting. The results showed that resveratrol markedly induced the phosphorylation of p38 
A

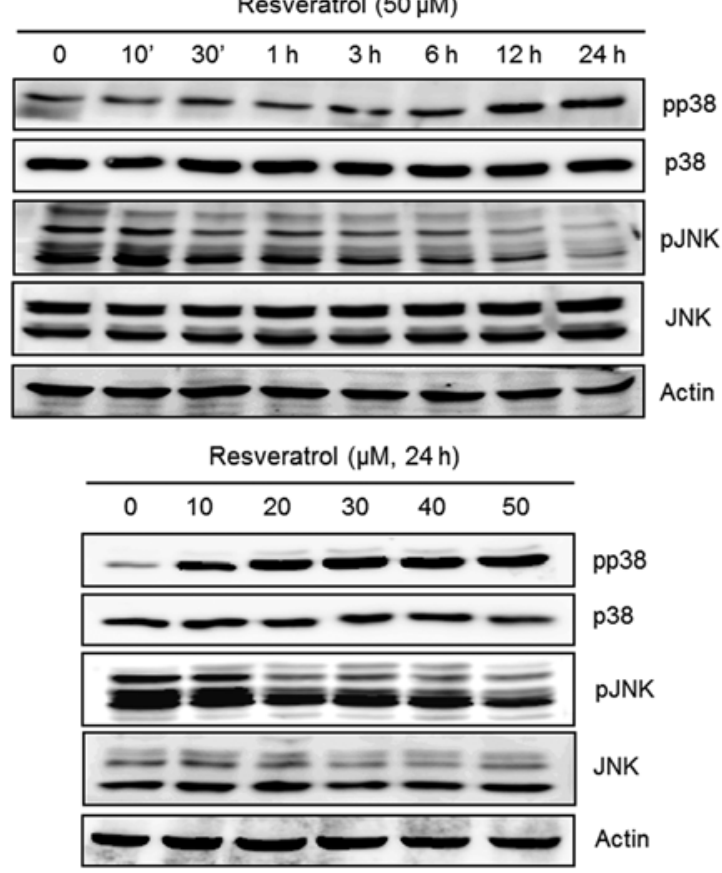

C

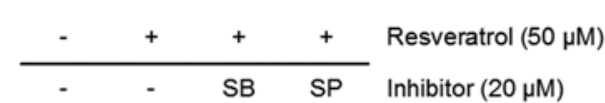

B

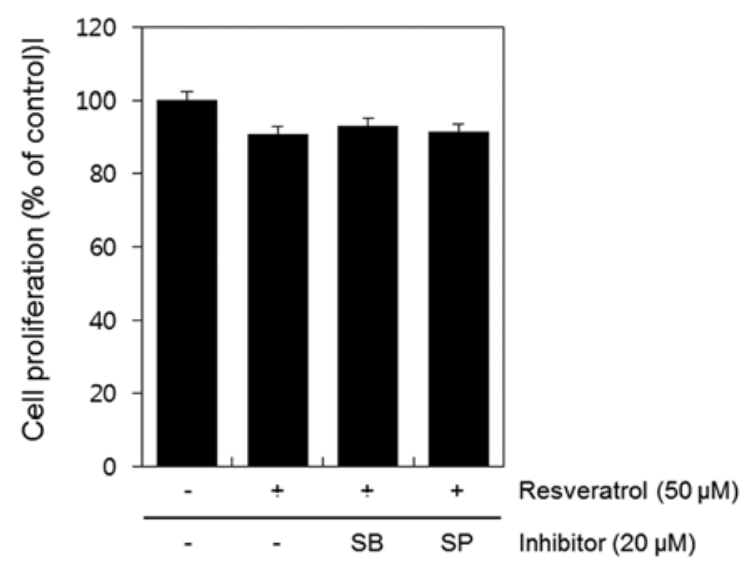

D

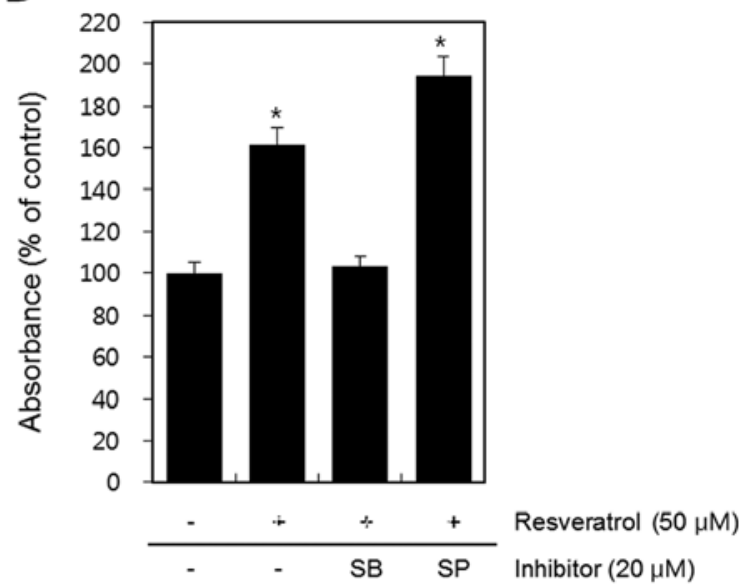

Figure 5. Effects of resveratrol on MMP-stimulated differentiation of HTB94 cells via the p38 kinase and JNK pathways. HTB94 cells were untreated (control) or treated with $50 \mu \mathrm{M}$ of resveratrol for the indicated time periods or with the indicated various concentrations of resveratrol for $24 \mathrm{~h}$. (A) Expression of pp38, p38, pJNK, JNK and actin was detected using western blot analysis. HTB94 cells were untreated (control) or treated with resveratrol in the absence or presence of $20 \mu \mathrm{M} \mathrm{SB} 203580$ (SB) or $20 \mu \mathrm{M} \mathrm{SP} 600125$ (SP) for $24 \mathrm{~h}$. (B) Cell proliferation was measured using MTT assay. (C) Activation of MMP-2 and MMP-9 was detected using gelatin zymography assay, and expression of type II collagen, SOX-9, pp38, p38, pJNK, JNK and actin was detected using western blot analysis. (D) Accumulation of sulfated proteoglycan was determined by Alcain blue staining. Statistically significant differences were noted between the control and the treatment groups. These data are the results of a typical experiment. ${ }^{*} \mathrm{P}<0.05$ compared to the control.

and reduced the phosphorylation of JNK in a time- and dosedependent manner (Fig. 5A). We left HTB94 cells untreated (control) or treated them with resveratrol in the absence or presence of $20 \mu \mathrm{M} \mathrm{SB} 203580$ or $20 \mu \mathrm{M} \mathrm{SP} 600125$ for $24 \mathrm{~h}$. To determine the effect of resveratrol on the viability of HTB94 cells, we performed an MTT assay. We found that co-treatment with resveratrol and SB203580 or SP600125 did not affect the viability of HTB94 cells (Fig. 5B). Next, activation of MMP-2 and MMP-9 was detected using gelatin zymography, and expression of type II collagen, SOX-9, pp38, pJNK and actin was assessed using western blotting. Inhibition of p38 kinase with SB203580 enhanced the effects of resveratrol on MMP-2 and MMP-9 expression, as well as its effects on the expression of type II collagen and SOX-9. Similar effects were observed upon co-treatment of cells with resveratrol and the JNK inhibitor SP600125 (Fig. 5C). Moreover, resveratrol-mediated production of sulfated proteoglycans, as assessed using Alcian blue staining, was suppressed in cells co-treated with the p38 kinase inhibitor SB203580 and resveratrol; inhibition of JNK with SP600125 in the presence of resveratrol further increased 
A

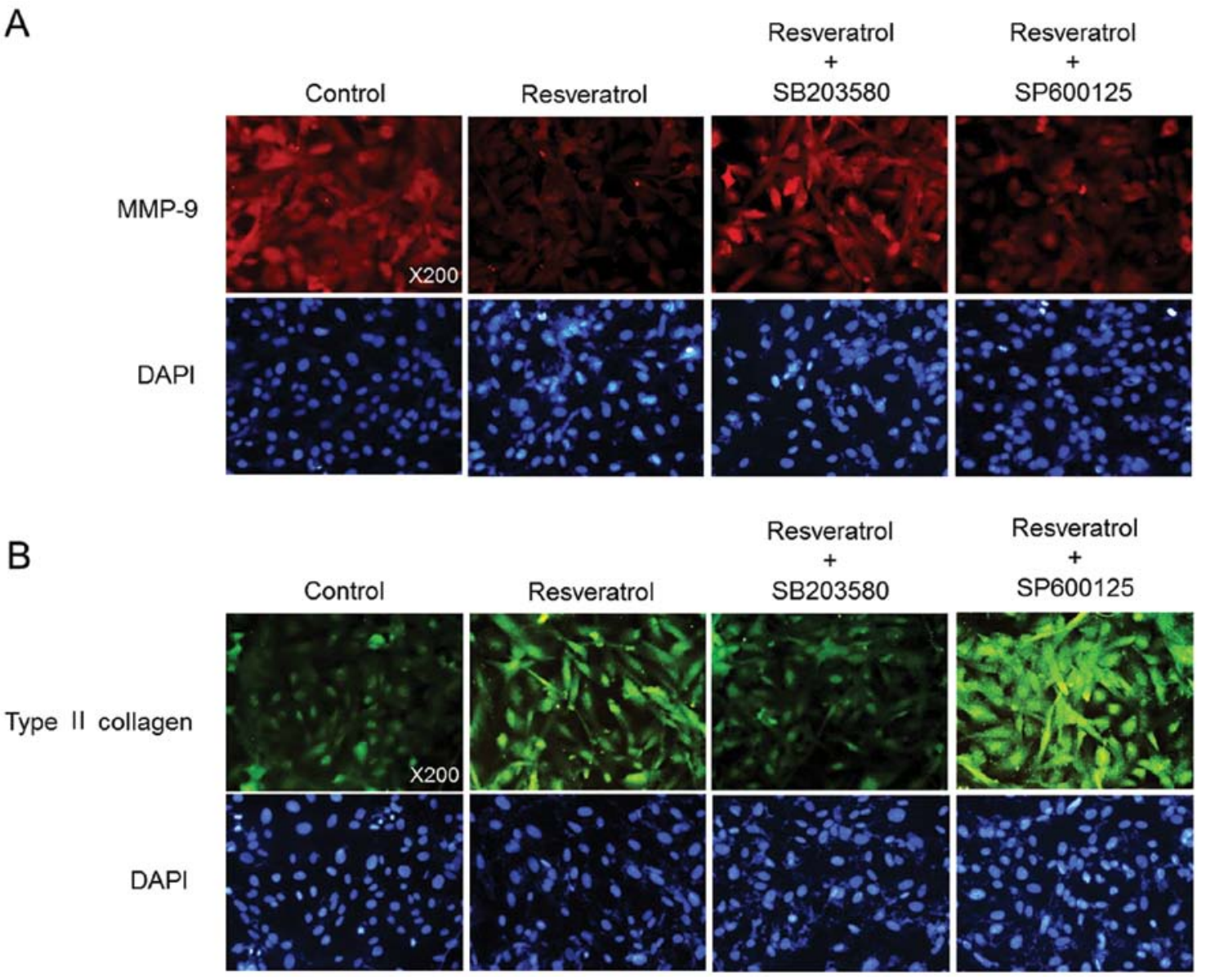

B

Figure 6. Effects of resveratrol on MMP-9-mediated regulation of type II collagen expression in HTB94 cells via the p38 kinase and JNK pathways. HTB94 cells were untreated (control) or treated with resveratrol in the absence or presence of $20 \mu \mathrm{M} \mathrm{SB} 203580$ or $20 \mu \mathrm{M} \mathrm{SP} 600125$ for $24 \mathrm{~h}$. (A and B) Expression of MMP-9 and type II collagen was detected by immunofluorescence staining. Statistically significant differences were noted between the control and the treatment groups. These data are the results of a typical experiment.

the resveratrol-induced production of sulfated proteoglycans (Fig. 5D). Moreover, consistent with the results of western blotting, the results of immunofluorescence analysis showed that inhibition of p38 kinase with SB203580 enhanced the suppression of MMP-9 by resveratrol and inhibited resveratrol-induced type II collagen expression (Fig. 6A). Inhibition of JNK kinase with SP600125 further increased the resveratrol-induced type II collagen expression (Fig. 6B).

Taken together, these results suggest that resveratrol attenuates MMP-regulated differentiation via the p38 kinase and JNK pathways in HTB94 chondrosarcoma cells.

\section{Discussion}

Resveratrol is a grape polyphenol, and is known to have anticancer, anti-oxidant, anti-inflammatory and neuroprotective activities. Resveratrol has been shown to prevent angiogenesis and cell migration in endothelial cells, and these properties of resveratrol make it a good candidate for use in the prevention of tumor progression (19). Resveratrol was recently shown to inhibit tumor cell adhesion to endothelial cells by blocking the expression of intracellular adhesion molecule-1 (20), and the resveratrol analog 4-[2-(3,5-dimethoxyphenyl)vinyl] pyridine was found to reduce the extent of differentiation of 3T3-L1 adipocytes (21).
Chondrosarcoma is a highly malignant cancer with high potential to invade locally and cause distant metastasis, with a predilection for metastasis to the lungs (22). Clinically, surgical resection remains the primary mode of treatment for chondrosarcoma. In the absence of effective adjuvant treatment, this mesenchymal malignancy has a poor prognosis, and therefore, it is important to explore novel and more effective therapeutic strategies (23). MMPs constitute a family of enzymes that enable degradation of cartilage. These proteolytic enzymes attack and degrade components of the ECM. Importantly, they contribute to the breakdown of type II collagen and other matrix proteins (4). Type II collagen is a major component of cartilage, where it is present at a high concentration that is essential for the functional maintenance of tissue (24). Moreover, in carcinomas, stromal cells participate in cancer progression by producing proteases such as MMPs. The expression of MMPs is a prognostic factor in human chondrosarcomas (25). Recent studies have demonstrated that endothelin-1 promotes MMP-13 production and migration in human chondrosarcoma cells through the focal adhesion kinase (FAK)/phosphatidylinositol 3-kinase (PI3K)/Akt/mammalian target of rapamycin (mTOR) pathways. These data suggest that endothelin-1 activates FAK/PI3K/AKT/mTOR signaling, which in turn activates I $\mathrm{B}$ kinase $\alpha / \beta$ and NF- $\kappa \mathrm{B}$, resulting in increased MMP-13 expression and the migration of human chondrosarcoma cells (26). 
Interleukin (IL)-1 $\beta$ induces MMP-13 expression by activation of the p38 MAPK/c-Fos/AP-1 and Janus kinase (JAK)/signal transducers and activators of transcription (STAT) pathways in chondrocytes. The p38 MAPK/c-Fos/AP-1 and JAK2/STAT1/2 pathways are involved in MMP-13 induction in IL-1 $\beta$-treated HTB94 human chondrocytes. Blocking these signaling pathways may have chondroprotective effects in cartilage degeneration (27). Another study demonstrated downregulation of MMP-13 by the root extract of Cyathula officinalis Kuan and its constituents in IL-1 $\beta$-treated chondrocytes. The results also showed that $C$. officinalis Kuan constituents may have the potential for conferring protection against cartilagedegrading disorders (28). Moreover, CCN3, a cysteine-rich protein family, enhanced the migration of chondrosarcoma cells by increasing MMP-13 expression via the $\alpha v \beta 3 / \alpha v \beta 5$ integrin receptor, FAK, PI3K, Akt, p65 and NF- $\mathrm{KB}$ signaltransduction pathways (29).

In the present study, we showed that resveratrol suppressed the activation of MMP-2 and MMP-9 in HTB94 human chondrosarcoma cells. Moreover, resveratrol induced the expression of type II collagen and SOX-9 and sulfated proteoglycan production. Inhibition of MMP-2 and MMP-9 with MMP inhibitor III further enhanced these effects of resveratrol. In addition, resveratrol markedly induced phosphorylation of p38 and reduced phosphorylation of JNK in HTB94 cells. Inhibition of p38 kinase with SB203580 enhanced resveratrolmediated suppression of MMP-2 and MMP-9 and inhibited resveratrol-induced type II collagen and SOX-9 expression. Inhibition of JNK with SP600125 decreased resveratrol-mediated suppression of MMP-2 and MMP-9, and further increased resveratrol-induced effects on type II collagen and SOX-9, and on the production of sulfated proteoglycans. In conclusion, the results of the present study suggest that resveratrol reduces the MMP-regulated differentiation of human chondrosarcoma cells via the p38 kinase and JNK pathways.

\section{Acknowledgements}

The present study was supported by a grant from the Korean Health Technology Research and the Development Project, Ministry of Health and Welfare, Republic of Korea (A1209601201-0000300).

\section{References}

1. Zyada MM and Shamaa AA: Is collagenase-3 (MMP-13) expression in chondrosarcoma of the jaws a true marker for tumor aggressiveness? Diagn Pathol 3: 26, 2008.

2. Tang CH and Tsai CC: CCL2 increases MMP-9 expression and cell motility in human chondrosarcoma cells via the Ras/Raf/ MEK/ERK/NF- $\mathrm{KB}$ signaling pathway. Biochem Pharmacol 83: 335-344, 2012

3. Power PF, Mak IW, Singh S, Popovic S, Gladdy R and Ghert M: ETV5 as a regulator of matrix metalloproteinase 2 in human chondrosarcoma. J Orthop Res 31: 493-501, 2013.

4. Lu YC, Jayakumar T, Duann YF, et al: Chondroprotective role of sesamol by inhibiting MMPs expression via retaining NF- $\kappa \mathrm{B}$ signaling in activated SW1353 cells. J Agric Food Chem 59: 4969-4978, 2011.

5. Im HJ, Li X, Chen D, et al: Biological effects of the plant-derived polyphenol resveratrol in human articular cartilage and chondrosarcoma cells. J Cell Physiol 227: 3488-3497, 2012.

6. Hidalgo $\mathrm{M}$ and Eckhardt SG: Development of matrix metalloproteinase inhibitors in cancer therapy. J Natl Cancer Inst 93: 178-193, 2001.
7. Abeles $\mathrm{AM}$ and Pillinger $\mathrm{MH}$ : The role of the synovial fibroblast in rheumatoid arthritis: cartilage destruction and the regulation of matrix metalloproteinases. Bull NYU Hosp Jt Dis 64: 20-24, 2006.

8. Tchetverikov I, Lohmander LS, Verzijl N, et al: MMP protein and activity levels in synovial fluid from patients with joint injury, inflammatory arthritis, and osteoarthritis. Ann Rheum Dis 64: 694-698, 2005.

9. Brisdelli F, D'Andrea G and Bozzi A: Resveratrol: a natural polyphenol with multiple chemopreventive properties. Curr Drug Metab 10: 530-546, 2009.

10. Lee SJ and Kim MM: Resveratrol with antioxidant activity inhibits matrix metalloproteinase via modulation of SIRT1 in human fibrosarcoma cells. Life Sci 88: 465-472, 2009.

11. Liu FC, Hung LF, Wu WL, et al: Chondroprotective effects and mechanisms of resveratrol in advanced glycation end productsstimulated chondrocytes. Arthritis Res Ther 12: R167, 2010.

12. Lee HS, Ha AW and Kim WK: Effect of resveratrol on the metastasis of $4 \mathrm{~T} 1$ mouse breast cancer cells in vitro and in vivo. Nutr Res Pract 6: 294-300, 2012.

13. Liu PL, Tsai JR, Charles AL, et al: Resveratrol inhibits human lung adenocarcinoma cell metastasis by suppressing heme oxygenase 1-mediated nuclear factor- $\kappa \mathrm{B}$ pathway and subsequently downregulating expression of matrix metalloproteinases. Mol Nutr Food Res 54 (Suppl 2): S196-S204, 2010.

14. Dhillon AS, Hagan S, Rath O and Kolch W: MAP kinase signalling pathways in cancer. Oncogene 26: 3279-3290, 2007.

15. Kim SJ, Ju JW, Oh CD, et al: ERK-1/2 and p38 kinase oppositely regulate nitric oxide-induced apoptosis of chondrocytes in association with $\mathrm{p53}$, caspase-3, and differentiation status. J Biol Chem 277: 1332-1339, 2002.

16. Cohen M, Meisser A, Haenggeli L and Bischof P: Involvement of MAPK pathway in TNF- $\alpha$-induced MMP-9 expression in human trophoblastic cells. Mol Hum Reprod 12: 225-232, 2006.

17. Kajanne R, Miettinen P, Mehlem A, et al: EGF-R regulates MMP function in fibroblasts through MAPK and AP-1 pathways. J Cell Physiol 212: 489-497, 2007.

18. Kim ES, Sohn YW and Moon A: TGF- $\beta$-induced transcriptional activation of MMP-2 is mediated by activating transcription factor (ATF) 2 in human breast epithelial cells. Cancer Lett 252: 147-156, 2007.

19. Azios NG and Dharmawardhane SF: Resveratrol and estradiol exert disparate effects on cell migration, cell surface actin structures, and focal adhesion assembly in MDA-MB-231 human breast cancer cells. Neoplasia 7: 128-140, 2005.

20. Park JS, Kim KM, Kim MH, et al: Resveratrol inhibits tumor cell adhesion to endothelial cells by blocking ICAM-1 expression. Anticancer Res 29: 355-362, 2009.

21. Hwang JT, Kim S, Choi I and Choi SY: Resveratrol analog 4-[2-(3,5-dimethoxyphenyl)vinyl]pyridine reduces differentiation of the 3T3-L1 adipocyte. Pharm Biol 51: 96-99, 2013.

22. Hou $\mathrm{CH}$, Chiang YC, Fong YC and Tang CH: WISP-1 increases MMP-2 expression and cell motility in human chondrosarcoma cells. Biochem Pharmacol 81: 1286-1295, 2011.

23. Tang $\mathrm{CH}$, Chen $\mathrm{CF}$, Chen WM and Fong YC: IL-6 increases MMP-13 expression and motility in human chondrosarcoma cells. J Biol Chem 286: 11056-11066, 2011.

24. Shinomura T, Ito K, Hook M and Kimura JH: A newly identified enhancer element responsible for type II collagen gene expression. J Biochem 152: 565-575, 2012.

25. Jawad MU, Garamszegi N, Garamszegi SP, et al: Matrix metalloproteinase 1: role in sarcoma biology. PLoS One 5: e14250, 2010.

26. Wu MH, Lo JF, Kuo CH, et al: Endothelin-1 promotes MMP-13 production and migration in human chondrosarcoma cells through FAK/PI3K/Akt/mTOR pathways. J Cell Physiol 227: 3016-3026, 2012.

27. Lim H and Kim HP: Matrix metalloproteinase-13 expression in IL-1 $\beta$-treated chondrocytes by activation of the p38 MAPK/c-Fos/AP-1 and JAK/STAT pathways. Arch Pharm Res 34: 109-117, 2011.

28. Park HY, Lim H, Kim HP and Kwon YS: Downregulation of matrix metalloproteinase-13 by the root extract of Cyathula officinalis Kuan and its constituents in IL-1 $\beta$-treated chondrocytes. Planta Med 77: 1528-1530, 2011.

29. Tzeng HE, Chen JC, Tsai CH, et al: $\mathrm{CCN} 3$ increases cell motility and MMP-13 expression in human chondrosarcoma through integrin-dependent pathway. J Cell Physiol 226: 3181-3189, 2011. 